\title{
Electrical Role for Severe Storm Tornadogenesis (and Modification)
}

\section{R.W. Armstrong ${ }^{1 *}$ and J.G. Glenn ${ }^{2}$}

${ }^{1}$ Department of Mechanical Engineering, University of Maryland, College Park, MD, USA

${ }^{2}$ Munitions Directorate, Eglin Air Force Base, FL, USA

\begin{abstract}
Damage from severe storms, particularly those involving significant lightning is increasing in the US. and abroad; and, increasingly, focus is on an electrical role for lightning in intra-cloud (IC) tornadogenesis. In the present report, emphasis is given to severe storm observations and especially to model descriptions relating to the subject.
\end{abstract}

Keywords: Tornadogenesis; Severe storms; Electrification; Lightning; Cloud seeding

\section{Introduction}

Benjamin Franklin would be understandably disappointed. Here we are, more than 260 years after first demonstration in Marly-la-Ville, France, of sparks drawn from a vertical iron rod in a thunderstorm [1], to establish the presence of lightning electrification and yet not having unraveled among the many complexities of variables involved in such severe weather condition a specific role, or not, for electrical aspects of the process of tornadogenesis. Bernard Vonnegut and colleagues performed pioneering researches to establish such connection during the period from the 1960s through the 1970s; see for example references [2-4]. It seems fair to say that a storm of investigations was spawned with results in opposition [5-7]. Davies-Jones RP [7] in particular led a valuable discussion $[8,9]$ ending with a concluding statement that "there [was] little observational evidence to support [the theory of a main role for thunderstorm electricity in tornadogenesis]". One purpose of the present report these many years afterward is to provide an update of experimental observations and analysis relating to the issue.

A second purpose of the present report is to carry on from an initiative begun with internal research funding at Eglin Air Force Base (AFB), FL, for the purpose of investigating the means by which a tornado could possibly be disrupted [10]. The reason for such funding, as will be demonstrated, was that a significant number of Air Force and other military bases were in the path of 'tornado alley', cutting a severe-storm-centered swath from the lower western portion of the US mid-west and tracking north by north-east to the Canadian border. Significant damage was shown to have occurred to military facilities located in the region, with accompanying loss of personnel, including within the civilian sector. Currently, the susceptible region has increased in size; and tornadic activity has also increased worldwide, as will be demonstrated in the following description. One outcome of the Eglin-funded research activity was a proposed focus on a role for intra-cloud (IC) lightning in tornadogenesis [11,12]. During the same period, the US National Academies had published a report in which attention was directed to a list of critical concerns in weather modification research among which items were 'cloud modeling issues' such as 'predictive models for severe weather events' and cloud model capability to track dispersion of seeding material both within and outside of seeded areas [13]. A later USNA outcome was the current federally-funded 2009-2017 VORTEX2 program 'to find previously unknown indications that a tornado will soon form' with report that the 'results could go beyond tornado forecasting' [14]. Also, a research session had been organized at the 2006 Annual Meeting of the American Association for the Advancement of Science (AAAS) [15]. In follow-up activity, a letter of support for needed research activity in weather science was submitted in response to solicitation from the National Science Board [16], and a note was published on the need for cooperation between weather modification practitioners and academic researchers dedicated to ameliorating the consequences of severe weather storms [17]. Need was expressed for interdisciplinary research on the topic relating to that recently touted for research in the broader field of meteorological sciences [18]. The present report continues in the same spirit of bringing in otherwise disparate subjects of electrical field equations, atomic-scale electron collisions, and cloud ionization/ ice nucleation chemistry aspects of cloud electrifications, all proposed to connect with important additional observations made in recent reports of tornadic activity within severe storm behaviors.

\section{Tornado Activities}

Figure 1 shows a distribution of AFBs within the central US taken to be 'at risk' or 'most at risk' from tornado damage, with inset information and a picture pertinent to devastation occurring at tornado-struck Tinker AFB. While recent attention has been given to the need for accurate assessment of cost estimations associated with extreme weather events [19], comparable damage to that indicated in Figure 1 occurred on April 26, 1991 at nearby McConnell AFB and spread through the surrounding area to produce 17 deaths and a determination of $\sim \$ 162 \mathrm{M}$ in damage to infrastructure. A storm at Oklahoma/Kansas on May 3, 1999, produced an estimation of $\sim \$ 1 B$ in damage. The AAAS meeting synopsis began with the statement: "If this nation had a foreign power do to us in one year what a devastating tornado can do to us in one day, we would use every means possible to fight it." [15] (Figure 1).

\section{Relation to US Severe Storm Activity}

The doubly-shaded geographical region covered in Figure 1 may be compared with the not-too-different similarly outlined area covered in Figure 2, as taken from a comprehensive environmental study of a number of severe storm systems reported by Carey and Buffalo [20].

*Corresponding author: RW Armstrong, Center for Engineering Concepts Development, University of Maryland, College Park, MD, 20742, USA, Tel: 1410 723 4616; E-mail: rona@umd.edu

Received August 10, 2015; Accepted August 31, 2015; Published September 14,2015

Citation: Armstrong RW, Glenn JG (2015) Electrical Role for Severe Storm Tornadogenesis (and Modification). J Climatol Weather Forecasting 3: 139. doi:10.4172/2332-2594.1000139

Copyright: (c) 2015 Armstrong RW, et al. This is an open-access article distributed under the terms of the Creative Commons Attribution License, which permits unrestricted use, distribution, and reproduction in any medium, provided the original author and source are credited. 


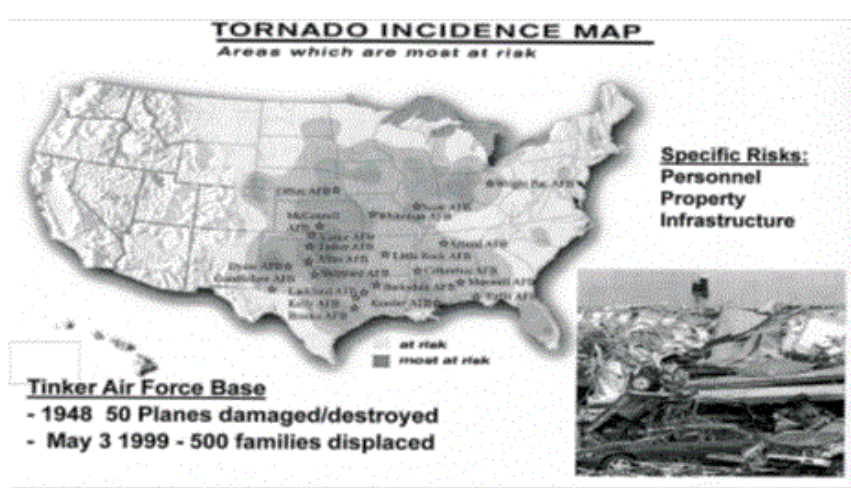

Figure 1: Distribution of US AFBs within the US particularly susceptible to severe weather damage.

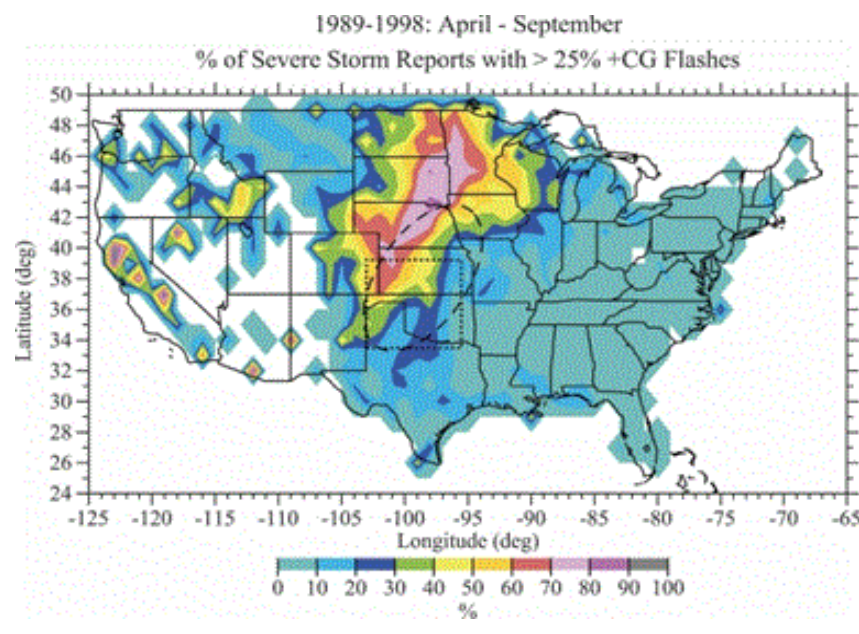

Figure 2: Severe storm percentages applicable to the IHOP_2002 and Carey and Buffalo study [20].

In Figure 2, the outline of dashed ellipsoidal and dotted square regions relate, respectively, to study of cloud-to-ground (CG) storm polarity results reported in a previous International $\mathrm{H}_{2} \mathrm{O}$ Project (IHOP_2002) and to that under current investigation. Comparison of Figures 1 and 2 shows that both Tinker and McConnell AFBs are positioned in the central region of both areas covered in the reported studies.

The main concern in [20] was relationship of CG polarity to cloud electrification and occurrence of lightning, specifically, to investigate the suggestion that strong wind updrafts with associated large liquid water contents in severe storms would cause an inverted lower cloud positive charge structure (changed from normally overwhelming negative charge) and lead to enhanced+CG lightning production. Such concern with CG lightning activity and strong updraft wind velocity relates to the model of tornadogenesis stemming from an important role for upward-deflected wind velocity occurring in the below cloud environment, quite different from the model description given in [12] that tornadogenesis occurs within the higher IC cloud environment consistent with the general observation that initiated tornadoes descend from above the lower cloud level. Nevertheless, Carey and Buffalo report a number of valuable observations/conclusions relating to their study of the structural, dynamical and microphysical details included in measurements both of a number of +CG and -CG severe storm behaviors. Their results for $+\mathrm{CG}$ activity were taken to 'support the hypothesis that broader, stronger updrafts and larger super cooled liquid water contents in the mixed-phase zone of convection cause the positive charging of graupel and hail via [collision-based noninductive charging] and the subsequent formation of a mid-level (i.e $-10^{\circ}$ to $-20^{\circ}$ C) positive charge region and enhanced production of -CG lightning ...'. Important issues of the magnitude of convective available potential energy (CAPE), cloud instability/buoyancy, lower cloud level (LCL), and low-level wind shear entered into the assessment of conditions for positive or (more prominent) negative storm activity. In summarizing description, Carey and Buffalo state: "Although the potential causative factors for both significant tornadoes and CG lightning polarity are many, complex, and the subject of current debate, our study in combination with recent studies on the environmental conditions associated with significant tornadic supercells may help explain the occasional coincidence between tornadogenesis and CG lightning polarity reversals." The LCL was concluded to be 'one of the best environmental discriminators between supercells that produce significant tornadoes (tornadic) and those that do not (non-tornadic). An LCL $<800 \mathrm{~m}$ was associated with significant tornadoes and $<1200$ $\mathrm{m}$ was associated with decreasing likelihood of significant tornadoes'. The latter LCL characterization is perhaps also to be associated with the Armstrong/Glenn (AG) emphasis given to an IC origin of tornadogenesis. (Figure 2)

\section{International Storm/Tornado Results}

Tornadic activity is 'popping up' with increasing frequency in other parts of the world, for example, as recently reported in detailed studies of such activity in South America, Europe and Asia [21-23]. Argentina has its own tornado alley in the La Pampa region where occurrences were compiled from newspaper accounts [21]. In the study, satellite and radar measurements were employed to monitor hail and, especially, lightning frequency as a proxy for intense and developing storms, reaching a not very high $\sim 0.3 \mathrm{~s}^{-1}$ flash rate in the mountainous region of the Argentina/Chile border, with reported tornadoes occurring further east in the central Pampas. Higher accounts of such flash rates in the US, both experimentally observed and employed in model calculations, are to be discussed. For a particular tornadic storm in Catalonia, at the Spanish east coast of the Iberian Peninsula extending into the Mediterranean Sea, detailed satellite and radar measurements of both CG and IC flash rates were analyzed for moderate lightning activity, heavy rainfall and a weak tornado inflicting minor ground damage [22]. A maximum IC lightning flash rate reaching $\sim 0.04 \mathrm{~s}^{-1}$ was measured just before tornado appearance and much smaller flash rate $<0.002$ $\mathrm{s}^{-1}$ for -CG updraft wind. Several features noted were a relatively low CAPE value, high precipitation, low buoyancy but no lightning jumps during the tornado. An initial sharp increase of the IC flash rate was associated with presumption of a stronger wind shear associated with the storm.

Of greater interest to our purpose is the case study presented for a microphysics analysis of a severe thunderstorm in India in which case the storm features were simulated with the Weather Research and Forecasting (WCF) model and compared with experimental observations and other model simulations [23]. Investigation of the storm focused on cloud-ice nucleation/generation among mixed phases in relation to CAPE, wind speed, maximum reflectivity, vertical velocity and the microphysics of latent energy release. The cloud ice and mixed phase processes were pointed out to be responsible for electrical activities inside the cloud. The storm intensity was taken to be especially enhanced by an increased updraft produced by greater latent energy effect of heating in the upper troposphere. 
Model equations were described for prediction of both mixing ratio and number concentrations of cloud hydrometeors spanning the conversion of water vapor into ice and mixed phases of snow and graupel, all within the convective cloud system. The vapor, moisture, ice and graupel content of the convective cloud system was shown to be an extremely important part of the severe storm problem. And this part of the complex interactive system was the subject of special study. In related investigation of the water-ice system, Marciante and Calvo [24] have recently asserted that 'Nucleation of water droplets from a supersaturated vapor is a basic atmospheric process involved in cloud, mist, and aerosol formation. The general understanding of nucleation through the seminal classical nucleation theory relies on equilibrium thermodynamic considerations. In practice, [the theory] contains a significant number of approximations in its evaluation of the critical nucleus, and often fails quantitatively against experiment or detailed numerical simulations if its ingredients are not known accurately enough'. Merciante and Calvo concentrate on the chemical physics of 'sticking' cross-sections of water molecules on cold sizeselected water clusters involving classical and quantum (path-integral) molecular dynamics simulations and provide comparison with experimental results. Earlier mention of Benjamin Franklin's surmised disappointment would no doubt be partly alleviated by such modern accumulation of knowledge that has been gained in the interim years of studying the complexity of microphysics within a severe storm system.

\section{Atmospheric Lightning}

Physics Today has recently reproduced the classic 1970 description given by Hans Panofsky of the global hydrodynamic and thermodynamic equations governing atmospheric behavior [25]. Four basic relations were presented: (1) an equation of state for dry air; (2) an equation for the thermodynamic system, including 'eddy' conduction and rate of energy addition by radiation; (3) continuity of material; and (4) Newton's $2^{\text {nd }}$ law for a rotating body. Inclusion of water vapor as the most important compositional variable was proposed to enter through the first two equations and with need for additional conservation equations. Attention was directed to interaction of the oceans and the atmosphere in influencing surface temperature patterns. Lightning was considered to be at a scale too small for consideration, as has been true until recent years in weather forecasting. Cloud seeding was mentioned as an example for mesoscale application of the equations and in relation to the as yet unknown effect on a hurricane.

A modern introduction to lightning beginning from the time of Benjamin Franklin is given in the recent book by Cooray [26]. In relation to the environmental reference made by Panofsky, Williams has produced an important review of lightning activity within the global electrical circuit [27]. Included topics were: (1) climate aspects of lightning and rainfall in the general convective circulation; (2) extremes in climate brought on by thunderstorms and lightning; (3) main temperature differential influences relating to cloud buoyancy; and, (4) the microphysics aspects of aerosols on cloud electrification. More extreme cloud systems were indicated to come for a warmer world but there is as yet no indication of enhanced mean thunderstorm lightning flash rates. In another report, Williams focused on further details of the physics of lightning by describing the 'polarity asymmetry' characteristics of positively and negatively charged flashes both in CG and IC occurrences, including in the latter case only production of gamma rays directed upward into space [28]. The observed asymmetries were taken to be associated with the microscopic asymmetry in mobility to be expected for free electrons and positive ions. Armstrong and Glenn employed such argument for the role of lightning-produced (electrons and) ions in tornadogenesis [12]. There is newspaper report of a rare form of 'dark lightning' associated with both gamma rays and $\mathrm{x}$-rays being generated by energetic electron collisions with atoms and molecules within storm clouds [29].

Evidence of variations in lightning character, both for IC and CG activities, was presented by Marshall and Stolzenburg [30] in a one-dimensional model description of changed electrostatic energy within a horizontally stratified cloud producing a 'spider type' flash occurrence. Focus was on the constraint provided by amount and (vertical) distribution of the charge structure. A voltage gradient of $\sim 10$ to $\sim 120 \mathrm{kV} / \mathrm{m}$ was taken to be typical within and below a typical storm cloud, compared to Vonnegut [3] employing a relatively low voltage gradient of $\sim 110 \mathrm{kV} / \mathrm{m}$ to obtain an order of magnitude estimation of a charge density of $\sim 5 \times 10^{3}$ elementary charges $/ \mathrm{cm}^{3}$ being associated with lightning production. So-called sprite production associated with Q-bursts was attributed to a cloud structure having an electrostatic energy of $\sim 10^{10} \mathrm{~J}$, say, compared to between $5 \times 10^{11}$ and $2 \times 10^{12} \mathrm{~J}$ within a mesoscale convective storm system, allowing a considerable number of (positive) lightning flashes in the range of $\sim 10^{7}$ to $10^{9} \mathrm{~J}$ in addition to the smaller number of energetic +CG flashes generating Q-bursts. The quantitative model calculations reported by Marshall and Stolzenburg were correlated with measurements obtained from balloons carrying electric field meters and meteorological radiosondes. The subject of electric discharges in the mesosphere was surveyed by Neubert, Rycroft, Farges, Blanc et al. with attention given to coordinated storm observations made in Southern Europe and including IC 'sprite' activity, ionization, electromagnetic radiation, and 'runaway' energetic electron actions [31]. Metzger and Nuss [32] have pointed more recently to the National Lightning Detection Network using sferics via low and verylow frequency radio bands to detect CG lightning strokes and timeof-arrival analysis with high and very-high frequency radio bands for such IC stroke detections. Several such systems were set up in Florida, Alabama and New Mexico. From such comprehensive measurements, the stroke or lightning flash detections were categorized according to accompanying severe wind or hail or mixed-type associations. A relative increase in CG over IC lightning activity occurred for onset of severe (ground level) wind behavior whereas the IC flash rate increased relatively over CG for hail type association and total lightning activity that always preceded the onset of severe weather. The preceding lightning behavior was taken to relate to the strength of updraft wind action as mentioned in numerous preceding studies.

Low frequency $(1 \mathrm{~Hz})$ infrasonic observations coordinated with Doppler radar measurements were reported by Bedard [33] in which a vortex whose maximum circulation was detected within an IC storm on 7 June 1995 in eastern Colorado and then was tracked on an eastwardly descending direction for approximately 30 minutes before evolving into a full-fledged tornado on the ground. The infrasonic method, that had been included in the mentioned survey by Neubert et al. [31] of electrical discharges in the mesosphere, provides for detection at distances up to $\sim 1000 \mathrm{~km}$, as also described by Bedard, and relates to monitoring of the acoustic energy contained in radial modes of vortex vibrations. Bedard employed results from the method also in several analyses of other archival observations of tornadoes. The infrasonic method is important because of being capable of providing evidence of otherwise unseen tornadogenesis being initiated within the IC structure. In a later report, Fierro et al. [34] outlined a test case of describing initiation of storm convection at a cloud-resolving scale via incorporation of total lightning data within a numerical weather research and forecasting (WRF) model, also done in conjunction with the Advanced Research WRF dynamic solver. Deep moist precipitating 
convection of water vapor and graupel was required to occur within the storm cloud structure in order to obtain agreement in the simulation of available measurements of storm parameters. Lightning flash rates from $0-0.7 \mathrm{~s}^{-1}$ were employed in the numerical calculations. The modeling of observations was judged to be promising. Of particular note was the observation that an incremental increase of water vapor at constant temperature in the vicinity of lightning regions led to acceleration of buoyancy and ultimately greater wind updraft in the modeled cloud behavior.

\section{Electrification Consequences of Lightning}

(Figure 3) Grigor'ev and Shiryaeva [35] have provided a modern account of lightning initiation and growth as critically-dependent on water droplets/hail/graupel in an IC atmosphere. The analysis may be compared with an earlier experimental report given by Watkins et al. [36] leading to a different conclusion of relative unimportance of electrical discharges for vortex stabilization within an atmosphere of only air. For the case considered by Grigor'ev and Shiryaeva, initiation of the lightning discharge was quantitatively assessed in terms of the instability of negatively charged micrometer-scale water drops and water-ice crystals within the IC environment. The model calculations may be seen to add to the (un-referenced) earlier model descriptions given by Vonnegut [2] but utilizing essentially the same electrification parameters. Among the modeled results are descriptions of the formation of an initial plasma region coupled with the development of calculations supporting effective auto-electron emissions during an electrical discharge in an avalanche-type mechanism (of $\sim 0.1 \mu \mathrm{s}$ duration) from electrically-unstable heavily charged water particles. Consideration of particle size dependencies was found to be relatively important with regard to establishment of the generated plasma and consequent activity of electron emissions, for example, covering unstable particle dimensions between $<0.3$ and $>30 \mu \mathrm{m}$ radii, much smaller than covered in Figure 3 taken from a compilation by Sin'kevich and Dovgalyuk [37] of critical electric field results.

The Figure 3 compilation of measurements for dependence on particle size of critical electric field strength $\mathrm{E}_{\mathrm{cr}}$, as reported by Sin'kevich and Dovgalyuk, provides a broad review of the same subject at the larger particle sizes considered by Vonnegut $[2,3]$ and included description of laboratory modeling of corona discharges. Focus was on $\mathrm{mm}$-scale ice crystals, pellets, and hail stone particles taken to initiate corona discharges and consequent intense ionization. The IC rate of

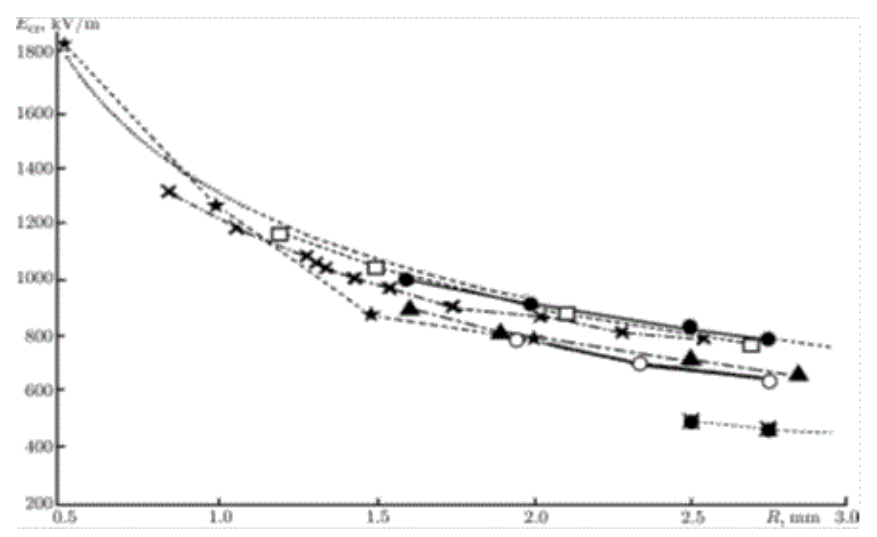

Figure 3: Compilation of critical electric-field strength, $E_{c r}$, measurements resulting in a corona discharge as dependent on droplet radius, following the relationship: Ecr [SGS units] $=1.5(\mathrm{Y}[\mathrm{dyn} / \mathrm{cm}] / \mathrm{R}[\mathrm{cm}])^{1 / 2}$. production of mainly negative ions during discharges was estimated to be $\sim 10^{7}$ per $\mathrm{cm}^{3} \mathrm{~s}^{-1}$ but otherwise had a charge sign dependence on the polarity of the external field. The discharge rate was taken to depend on the intensity of IC precipitation and when very high, the corresponding ion formation rate was proposed to increase by several orders of magnitude. Important 'characteristics of the processes of hydrometeor charging' involved particle collisions and break-ups. And in further relationship to the subject, Arseniev and Shelkovnikov [38] have provided a magnetohydrodynamic description of the field equations both for magnetic and electric characterization of a stable tornado. Excellent agreement was obtained between calculations of the wind velocity dependence on distance from the tornado core and reported measurements made for the devastating May 3, 1999, tornado in Oklahoma, already mentioned in regard to Figure 1. A matching calculation was made for the electric field dependence. Other agreement was described with observations of horizontal lightning occurring in the cores of tornadoes in Nebraska and in Kansas. As will be discussed, Rathbun [39] appears to have been first in pointing to the accompanying magnetic characteristics of tornadogenesis within a thunderstorm. (Figure 4)

The role of water vapor, water droplets, ice crystals, and graupel are all important to lightning generation and possible tornadogenesis in the IC environment. Armstrong and Glenn [12] sought to establish an important role for smaller particle lightning-induced ionization in vortex development through addition of such consideration to Vonnegut's focus on cloud electrification. Figure 4 is presented to show that such effect is important. In the figure, a closed diamond point is shown for Rathbun's description of tornadogenesis associated with CG lightning strikes, involving $\sim 10^{10}$ positive ions $/ \mathrm{cm}^{3}$ being created and driven upward to lower cloud positive charge [39]; see the previous estimations made by Wilkins [5]. The hydrogen radius of $\sim 10^{-15} \mathrm{~m}$ is taken from Arrington and Sick [40] although perhaps a larger size should be taken as reflective of the distance of influence. The closed circle point applies for Vonnegut's concern with micron-scale and larger particles within a cloud volume containing $2 \mathrm{~g} / \mathrm{m}^{3}$ water concentration and the linear dependence is his predicted relationship for dependence on particle size, including the largest open-circle particle for graupel; see http://wrh.noaa.gov/Flagstaff/science/cloud. $\mathrm{htm}$ for modern description of a typical moist cloud containing $\sim 0.5$ $\mathrm{g} / \mathrm{m}^{3}$ of water. Dashed lines show the dependencies for smaller and larger water contents. The top-most filled square point applies for $100 \%$ ionization of the corresponding $\sim 6.7 \times 10^{23} \mathrm{OH}^{-}$concentration/ $\mathrm{m}^{3}$ driven upward in the IC environment to promote updraft wind and eventual molecular recombination. The appearance of the graph is relatively unchanged if the abscissa scale is changed to a plot on the basis of particle mass. The ionization consideration relates reasonably directly to the description given by Vonnegut [3] and Sin'kevich and Dovgalyuk [37]. An example of comparative precipitation charge, from 2 to $220 \mathrm{pC}$, and particle size, from 0.6 to $3.8 \mathrm{~mm}$, measurements have been reported by Bateman, Marshall, Stolzenburg and Rust [1999] for a thunderstorm in New Mexico [41].

\section{Water Chemistry and Ionization Measurements}

A comprehensive review of the chemistry of water and ice has been given by Huang et al. [42]. In many ways, the behavior of the material is described as anomalous and the review attempts to deal with a number of the anomalies such as the influence of the $\mathrm{H}-\mathrm{O}$ bond relaxation on melting point, the "supersolid skin" of ice that is responsible for slipperiness, and the bi-phase structure of nano-droplets and nano-ice. For instance, such water nano-droplets undergo significant melting 


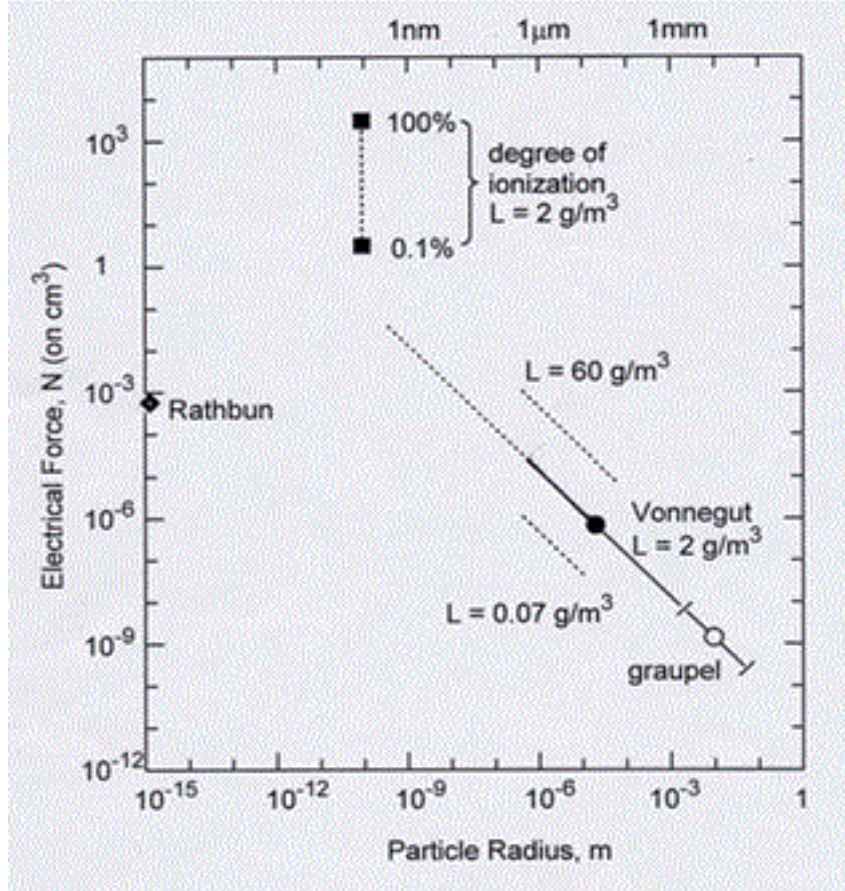

Figure 4: Electrical force vs. particle size for important influence of ionization.

point elevation and freezing point depression. It is well known that water has a relatively high surface free energy that plays an important role in nucleation of its phase transformation behavior. A much earlier description had been given by Turnbull and Vonnegut [43] of nucleation catalysis theory relating, for example, to a role for coherent nucleation of ice on silver iodide particles, a favorite material for "cloud seeding". Armstrong and Glenn have proposed cloud seeding of the super-saturated storm clouds in order to prevent tornadogenesis [12]. The subject of nucleation has recently been reviewed by Sear [44], particularly including latest results on nucleation and growth of ice crystals. The subject of non-classical nucleation was given emphasis, particularly including latest results on special difficulty experienced with computer simulation of the process.

Olofsson and Hepler [45] had earlier established an enthalpy of reaction for ionization of aqueous $\mathrm{H}_{2} \mathrm{O}$ of $55.815 \mathrm{~kJ} / \mathrm{mole}$ and the National Institute of Standards and Technology (NIST) listing for gaseous ionization is $\sim 1633 \mathrm{~kJ} / \mathrm{mole}$. Both energies seem relatively easy to be supplied at the temperatures of the order of $\sim 30,000 \mathrm{~K}$ achieved in a lightning discharge and consequent duration. An early report on ionization of water vapor by impact of slow electrons was given by Smyth and Mueller [46]. Bandura and lvov [47] have given a semi-theoretical analysis based on thermodynamics description of the ionization constant for liquid water that involves two molecules yielding $\mathrm{H}_{3} \mathrm{O}^{+}$and $\mathrm{OH}^{-}$. Quite interestingly, the authors state that "Because the proton hydration is completed under all conditions of practical interest, we assume that this [two molecule] reaction may be used as a representative model for both liquid and vapor phases up to the zero density limit." Naturally, the reported reaction rate constant was shown to increase strongly at lower density and higher temperature. The preceding results may be compared with the model simulation results obtained by Huthmacher et al. [48] of low energy electron dynamics $(<100 \mathrm{eV})$ achieved in liquid water by laser irradiation. The generation of free electrons caused impact ionization and a large additional amount of secondary electrons. More extreme description of waterbased atmospheric plasma generation in ball lightning discharges has been described by Dubrowsky et al. [49], including mass spectroscopy measurements of charged water, ammonia and nitrous oxide molecules Vonnegut [2]. An earlier report preceding that of Huthmacher et al. was made of such synthesized ball lightning 'fireballs' generated by burning of silicon nanoparticles emitting electrons at $1000 \mathrm{~K}$, not quite the higher temperature associated with a lightning strike [50]. At the top cloud height, there have been reported observations of 'blue jets', that are narrow cones of the colored light propagating upward at speeds of $\sim 100 \mathrm{~km} / \mathrm{s}$, for example, as investigated in detail by Wescott et al. [51], and perhaps not unrelated to the occurrence of luminosity and blue light described within the core of a number of previously described tornado behaviors $[2,38]$. Wescott et al. reported a peak in IC stroke emissions at the time of occurrence of a strong updraft in wind velocity and significant ionization. Other description of ionization and dissociation of condensed molecules on IC ice-grain surfaces has been reported for high energy cosmic rays and simulated by bombardment of heavy ions [52]. Positive and negative ions ejected by impact from ice surfaces were analyzed by means of mass spectroscopy.

\section{IC Lightning and Tornadogenesis}

One example of an alternative model description of tornado development within a supercell storm involves strong ground-level wind shear that tilts upward under appropriate condition to potentially produce multiple vortices surrounding a central circulation center [53]. The emphasis is on a continuous supply of lifted moisture inflow into a tornadic updraft from near ground level otherwise relying on a hydrodynamic-type description of CG wind velocity and cloud mechanics in the global manner outlined more broadly by Panofsky [25]. No obvious role of cloud electrification would be involved in the generation of these type tornadoes. More recently, a primary role for electrical force was brought in as a facilitator of IC tornadogenesis by Patton, Bothun and Sessions [54] with focus on the fact that many tornadic vortices begin at storm mid-level and build downward into ground level tornadoes, more or less in the same manner as originally described by Vonnegut $[2,3]$. Important connection was established with conditions of high wind shear and large CAPE, in the latter case as already mentioned for the description given in the detailed analysis reported by Carey and Buffalo [20]. In the Patton et al. model, a midlevel embryonic tornado structure, enhanced by electrical action, is taken to lead to a central downdraft that acts to build the vortex rotation in a downward direction. Historical electrical observations, even predating Benjamin Franklin, are quoted. Charge layer stratification in a cylindrically rotating system is an essential element of the model and local perturbation caused by downdraft or electromagnetic energy reduction is proposed to lead to cylindrical polarity with central negative charge. Latent heat from freezing provides buoyancy within the system. Reasonable negative core charge densities associated with $\mathrm{mm}$ size particles are described in the accompanying calculations; for example, a critical threshold core charge density $\left[>\sim 5 \mathrm{nC} / \mathrm{m}^{3}\right]$ was estimated to lead with high probability to tornadogenesis and its maintenance on a time scale of a few minutes.

Armstrong and Glenn (AG) have given emphasis in their $\mathrm{H}_{2} \mathrm{O}$ based model description of tornadogenesis to the role of lightning in generating $\mathrm{OH}^{-}$and $\mathrm{H}^{+}$particle densities driven in respective opposite up and down directions to provide wind enhancement and recombination energies for tornadic activity [11,12]; see Figure 3. A relevant reference on the issue is the reported study involving lightning and Doppler radar observations of two tornadic storms in Oklahoma in 
1981 [55]. Emphasis was given to one of the storms reaching a peak in the IC lightning flash rate of $\sim 0.3 \mathrm{~s}^{-1}$ at a height of $6 \mathrm{~km}$ and occurring in association with build-up of cyclonic shear at an LCL height of 1.5 $\mathrm{km}$. The obtained measurements and referenced others were taken "to suggest that 1 ) most tornadic storms ( $80 \%$ or more) have an increase in total flash rates near the time of the tornado, and 2) the increase in total flash rates is often dominated by intracloud flashes". We suggest that the flash rate may be taken to be roughly analogous to the mechanical example of an initial push rate being required to generate a spinning top; and, the need for continued pushing for spin enhancement relates to Vonnegut's admission of needed additional lightning flash enhancement to sustain a tornado [2,3]. Figure 5 shows higher reported lightning flash rate results for a May 221997 severe storm near Orlando FL [56]. Williams et al. [56] and later Buechler et al. [57] had reported far greater IC flash rates as compared with CG values preceding tornadogenesis. In the first case, Williams et al. reported IC flash rate 'jumps' of 3 to $8 \mathrm{~s}^{-1}$, far larger than mentioned above, that were associated with follow-on sudden increases in ground level wind velocities of 50 to $70 \mathrm{mph}$. Very significantly, the wind velocity buildups were found to lag behind the flash rate jumps by 5 to 20 minutes. The results provide evidence for the AG model consideration that enhancement of wind velocity should follow the flash rate influence on ionization [11]. Anderson and Freier [58] have describe an asymmetry character for the CG generated positive or negative rates of charging while pointing out that positive lightning discharges release much more energy and that CG negative discharges remove only a small fraction of the total LCL negative charge. The authors point to the net charge level in a thunderstorm cloud being much greater than can be transformed by most lightning discharges. Comparison of the discussion given by Anderson and Freier may be made with the AG account for reduction of the LCL charge level through IC positive charge driven downward for recombination and consequent energy release. (Figure 5)

Williams et al. [59] provided an important assessment of atmospheric thermodynamics and electrical conditions favorable to the several conditions of thunderstorm updraft, its associated microphysics and the lightning flash rate. A tropical system was investigated with follow-on extension of the results to continental US consideration. Effects of temperature gradient, cloud water content and updraft velocity were described, particularly with concern for the lightning flash rate as a function of cloud height. Very interestingly, a Figure (4c) was presented of compiled CG and IC/CG positive lightning flash locations centered on the same US tornado incidence and severe storm coverages shown here in Figures 1 and 2. Very recently, Nishihashi et al. [60] have reported on both CG and IC lightning activity in connection with tornadogenesis occurring on 2 September 2013 on the Kanto Plain in Japan. Their measurements included observations made by the (Japan) Lightning Detection Network (LIDEN), coupled with radar reflectivity and Doppler velocity data. Rapid increases in both CG and total flash rates, comparable to those observed in the US, were

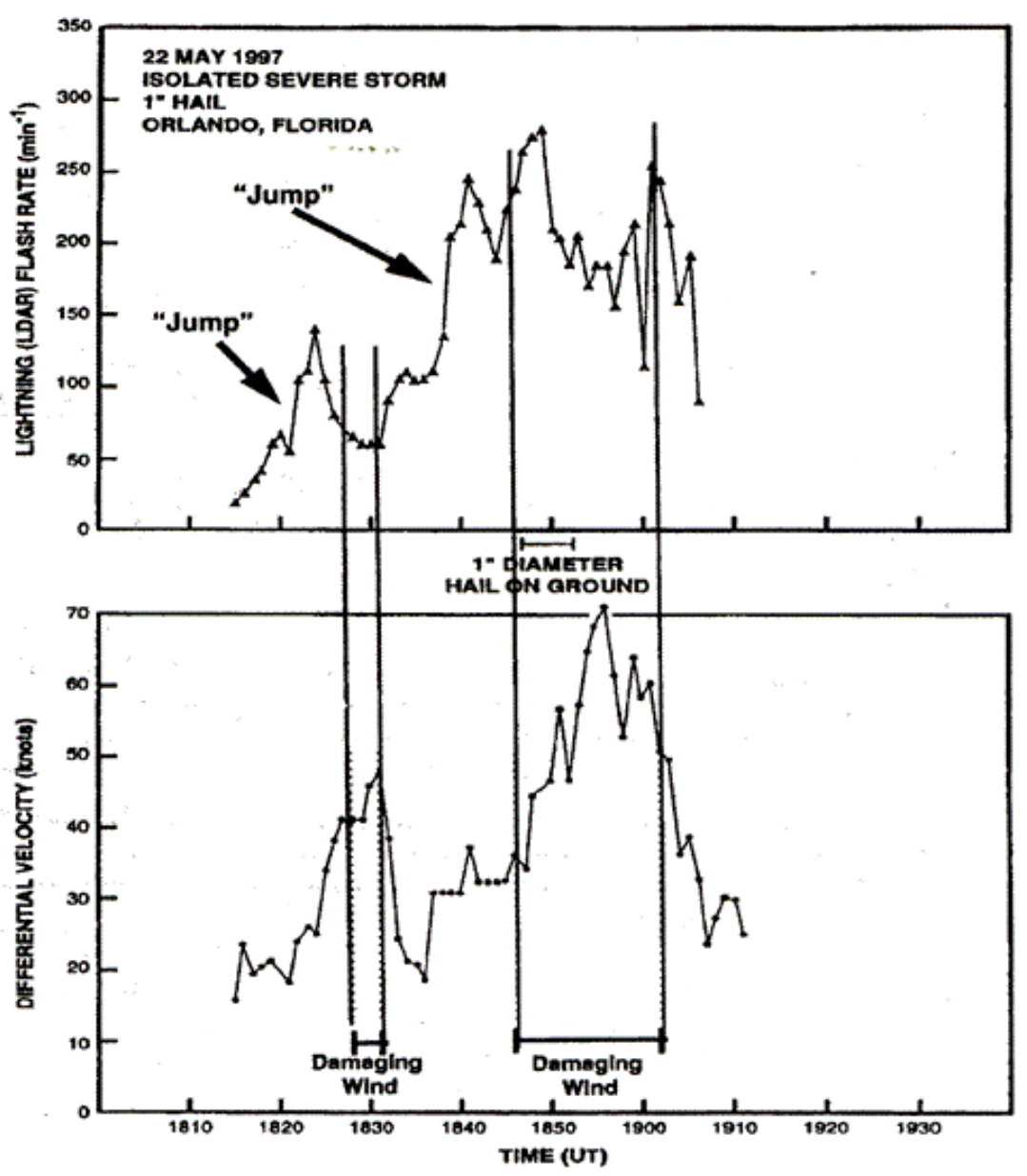

Figure 5: Correlation of reported lightning flash rate and ground level wind velocity [56]. 
Citation: Armstrong RW, Glenn JG (2015) Electrical Role for Severe Storm Tornadogenesis (and Modification). J Climatol Weather Forecasting 3: 139. doi:10.4172/2332-2594.1000139

recorded before tornadogenesis. Agreement was expressed with the results of Williams et al. [56] in that the lightning jumps were observed minutes before tornadogenesis. The CG flashes were essentially totally negative (96\%). A reason suggested for the lightning jumps was updraft enhancement above the cloud freezing level caused by larger vorticity of the mesocyclone in the mid-cloud region compared to that at LCL.

It was suggested by Nishihashi et al. that detection of such rapid flash rate occurrences could be employed on a severe weather warning decision-making basis. In the US, Strader and Ashley [61] employed the national lightning detection network (NLDN), along with geographic information system techniques, to assess the relationship only of CG lightning and severe weather development. It was concluded that knowledge of the lightning flashes alone was not sufficient in themselves to detection of a 'jump' prior to tornadogenesis. Rudlosky and Fuelberg [62] have reported recently also on lightning detection for storm severity via the Automated Warning decision Support System (WDSS) and have given a description of correlation procedures for analysis of severe storm conditions. Documentation of more than 1200 severe and non-severe storms in the US mid-Atlantic region was achieved. It was concluded that tornadic storms exhibited much greater IC and CG flash rates. McCaul and Cohen in an earlier report [63] had contributed the important finding that deep IC moisture layers, at least $1.5-2.0 \mathrm{~km}$ thick, proved helpful to 'maximizing updraft overturning efficiency' and increasing the chances of large hail, frequent lightning and heavy precipitation. The authors discuss the importance of buoyancy with an updraft of $\sim 55 \mathrm{~m} / \mathrm{s}$ for a CAPE value of $2000 \mathrm{~J} / \mathrm{kg}$. The observation relates to the AG suggestion that intentional cloud seeding of the supersaturated IC structure would help to prevent tornadogenesis in favor of increased precipitation, and even should possibly involve research on seeding of appropriately charged particles [11].

\section{Summary}

Surely, Benjamin Franklin would understandably be a bit discouraged, at least at first, because we are not further along in understanding tornadogenesis; and he would be especially downhearted that a role for lightning has not been quantitatively determined. But on the other hand, undoubtedly he would be impressed by the accumulated knowledge obtained on the many multi-scale severe storm parameters that are now understood each in its own way to play a role in tornadogenesis, and maybe with several more parameters to be specified. The topic deserves more attention in order to prevent severe storm damage [17]. In the present report, focus has been on IC lightning for embryonic tornadogenesis within the complex internal cloud structure. Issues of electrical polarity characteristics, CAPE, cloud buoyancy and positioning, all phases of water structure, and especially including the micro-physics of ionization and plasma generation, are proposed to enter relevantly into the needed comprehensive description of severe storm behavior and tornadogenesis yet to come.

\section{Acknowledgements}

Appreciation is expressed for initial Entrepreneurial Research Funding awarded to JGG at Eglin Air Force Base, FL; and then, for Eglin AFB Contract FA8651-04-D-0159 awarded to RWA. Also CM Lindsay, Eglin AFB, is thanked for recent provision of information on reference data.

\section{References}

1. Krider EP (2006) Benjamin Franklin and lightning rods. Physics Today 59: 4248.

2. Vonnegut B (1960) Electrical theory of tornadoes. Journal of Geophysical Research 65: 203-212.
3. Vonnegut B (1963) Some facts and speculations concerning the origin and role of thunderstorm electricity. Meteorological Monographs 5: 224-240.

4. Ryan RT, Vonnegut B (1971) Formation of a vortex by an elevated electrical heat source. Nature Physical Science 233: 142-143.

5. Wilkins EM (1964) The role of electrical phenomena associated with tornadoes. Journal of Geophysical Research 69: 2435-2447.

6. Wilkins EM, McConnell LT (1968) Threshold conditions for vortex-stabilized electrical discharges in the atmosphere. Journal of Geophysical Research 73: 2559-2568.

7. Davies-Jones RP, Golden JH (1975) On the relation of electrical activity to tornadoes. Journal of Geophysical Research 80: 1614-1616.

8. Vonnegut B (1975) Comment on 'On the relation of electrical activity to tornadoes' by RP Davies-Jones and JH Golden'. Journal of Geophysical Research 80: 4559-4560.

9. Davies-Jones RP, Golden JH (1975) Reply. Journal of Geophysical Research 80: 4561-4562.

10. Glenn JG (2001) Disruption of a vortex. Entrepreneurial Research Funding 2303PM06, AFRL/MN, Eglin Air Force Base, FL.

11. Armstrong RW, Glenn JG (2005) Role of intracloud lightning in tornadogenesis. Report AFRL-MN-EG-TR-2005-7025, Air Force Research Laboratory, Munitions Directorate, Eglin Air Force Base.

12. Armstrong RW, Glenn JG (2006) Role for lightning in tornadogenesis and possible modification, Journal of Weather Modification 38: 77-81.

13. The National Academies (2003) Report In Brief: Critical Issues in Weather Modification Research. National Academies Press, Washington, D.C.

14. The Washington Post (2012) Data may show why tornadoes emerge. Tuesday, May 1, 2012.

15. Glenn JG, Armstrong RW, Bruintjes R (2006) Preventing Severe Storm Damage. $172^{\text {nd }}$ National Meeting of the American Association for the Advancement of Science 70-71.

16. Armstrong RW, Alamaro M, Glenn JG (2006) Public Comment, Nationa Science Board, National Science Foundation, Hurricane Warning: The Critical Need for a National Hurricane Research Initiative, 27 October.

17. Armstrong RW, Glenn JG, Alamaro M (2007) Coordination, research needed in weather science. Physics Today 60: 10-12.

18. Goodsite M, Gammelgaard A, Culton J, Gross A, Holm P (2012) Interdisciplinary research in meterorological sciences. Climatology \& Weather Forecasting 1: e101.

19. Jenkins K (2013) Extreme weather and climate change cost assessments. Climatology \& Weather Forecasting 1: e104

20. Carey LD, Buffalo KM (2007) Environmental control of cloud-to-ground lightning polarity in severe storms Monthly Weather Review 135: 1327-1353.

21. Rasmussen KL, Zuluaga MD, Houze Jr RA (2014) Severe convection and lightning in subtropical South America. Geophysical Research Letters 41 7359-7366.

22. Bech J, Arúsb J, Castán S, Pineda N (2015) A study of the 21 March 2012 tornadic quasi linear convective system in Catalonia. Atmospheric Research 158: $192-209$

23. Halder M, Hazra A, Mukhopadhyay P, Singh D (2015) Effect of the bette representation of the cloud ice-nucleation in WRF microphysics schemes: $A$ case study of a severe storm in India. Atmospheric Research 154: 155-174.

24. Marciante M, Calvo F (2015) Communication: Nucleation of water on ice nanograins: Size, charge, and quantum effects. Journal of Chemical Physics 142: 171104.

25. Panofsky H (2014) Physics and Environment: Analyzing atmospheric behavior Physics Today 67(6): 38-41.

26. Cooray, Vernon (2015) An Introduction to Lightning (1 $\left.{ }^{\text {st }} e d n\right)$. Springer Publisher Berlin.

27. Williams ER (2005) Lightning and climate: A review. Atmospheric Research 76: $272-287$.

28. Williams ER (2006) Problems in lightning physics-the role of polarity asymmetry Plasma Sources Science and Technology 15: 91-108. 
Citation: Armstrong RW, Glenn JG (2015) Electrical Role for Severe Storm Tornadogenesis (and Modification). J Climatol Weather Forecasting 3: 139. doi:10.4172/2332-2594.1000139

29. The Washington Post (2013) 'Dark lightning' might carry a lifetime's worth of damaging radiation. Tuesday, April 23.

30. Marshall TC, Stolzenburg M (2002) Electrical energy constraints on lightning Journal of Geophysical Research 107

31. Neubert T, Rycroft M, Farges T, Blanc E, Arnold N, et al. (2008) Recent results from studies of electric discharges in the mesosphere. Surveys in Geophysics 29: 71-137.

32. Metzger E, Nuss WA (2013) The relationship between total cloud lightning behavior and radar-derived thunderstorm structure. Weather and Forecasting 28: 237-253

33. Bedard AJ (2005) Low-frequency atmospheric acoustic energy associated with vortices produced by thunderstorms. Monthly Weather Review 133: 241-263.

34. Fierro AO, Mansell ER, Ziegler CL, MacGorman DR (2012) Application of a lightning data assimilation technique in the WRF-ARW model at cloud-resolving scales for the tornado outbreak of 24 May 2011. Monthly Weather Review 140: 2609-2627.

35. Grigor'ev Al, Shiryaeva SO (1996) The possible physical mechanism of initiation and growth of lightning. Physica Scripta 54: 660-666

36. Watkins DC, Cobine JD, Vonnegut B (1978) Electric discharges inside of tornadoes. Science 199: 171-174

37. Sin'kevich A, Dovgalyuk Yu A (2013) Corona discharge in clouds. Radiophysics and Quantum Electronics 56: 11-12.

38. Arseniev SA, Shelkovnikov NK (2012) Electromagnetic fields in tornados and spouts. Moscow University Physics Bulletin 67: 290-295.

39. Rathbun ER (1960) An electromagnetic basis for the initiation of a tornado. Journal of Meteorology 17: 371-373.

40. Arrington J, Sick I (2015) Evaluation of the proton charge radius from electronproton scattering. Journal of Physical and Chemical Reference Data 44

41. Bateman MG, Marshall TC, Stolzenburg M, Rust WD (1999) Precipitation charge and size measurements inside a New Mexico mountain thunderstorm. Journal of Geophysical Research 104: 9643-9653.

42. Huang Y, Zhang X, Ma Z, Zhou Y, Zheng W, et al. (2015) Review: Hydrogenbond relaxation dynamics: Resolving mysteries of water ice. Coordination Chemistry Reviews 285: 109-165

43. Turnbull D, Vonnegut B (1952) Nucleation catalysis. Industrial and Engineering Chemistry 44: 1292-1298.

44. Sear RP (2012) The non-classical nucleation of crystals: microscopic mechanisms and applications to molecular crystals, ice and calcium carbonate. International Materials Reviews 57: 328-356.

45. Olofsson G, Hepler LG (1975) Thermodynamics of ionization of water over wide ranges of temperature and pressure. Journal of Solution Chemistry 4: 127-143.

46. Smyth HD, Mueller DW (1933) The ionization of water vapor by electron impact. Physical Review 43: 116-120

47. Bandura AV, Lvov SN (2006) The ionization constant of water over wide ranges of temperature and density. Journal of Physical and Chemical Reference Data 35: $15-30$

Citation: Armstrong RW, Glenn JG (2015) Electrical Role for Severe Storm Tornadogenesis (and Modification). J Climatol Weather Forecasting 3: 139. doi:10.4172/2332-2594.1000139
48. Huthmacher K, Herzwurm A, Gnewuch M, RitterK, Rethfeld B (2015) Monte Carlo simulation of electron dynamics in liquid water. Physica A 429: 242-251.

49. Dubowsky SE, Friday DM, Peters KC, Zhao Z, Perry RH, et al. (2015) Mass spectrometry of atmospheric-pressure ball plasmoids. International Journal of Mass Spectrometry 376: 39-45.

50. Jermey N. A. Matthews (2008) Nanoparticles in ball lightning, Physics Today 61: 18.

51. Wescott EM, Sentman DD, Heavner MJ, Hampton DL, Vaughan Jr OH (1998) Blue jets: their relationship to lightning and very large hail fall and their physical mechanisms for their production. Journal of Atmospheric and Solar Terrestrial Physics 59: 713-724.

52. Martinez R, Bordalo V, da Silveira EF, Boechat-Roberty HM (2014) Production of $\mathrm{NH} 4+$ and $\mathrm{OCN}$ - ions by the Interaction of heavy-ion cosmic rays with $\mathrm{CO}$ $\mathrm{NH} 3$ interstellar ice. Monthly Notices of the Royal Astronomical Society 444: 3317-3327.

53. Klemp JB, Rotunno R (1983) A study of the tornadic region within a supercell thunderstorm. American Meteorological Society 359-377.

54. Patton FS, Bothun GD, Sessions SL (2008) An electric force facilitator in descending vortex tornadogenesis. Journal of Geophysical Research 113

55. MacGorman DR, Burgess DW, Mazur V, Rust WD, Taylor WL, et al. (1989) Lightning rates relative to tornadic storm evolution on 22 May 1981. Journal of Atmospheric Science 46: 221-250.

56. Williams E, Boldi B, Matlin A, Weber M, Hodanish S, et al. (1999) The behavior of total lightning in severe Florida thunderstorms. Journal of Atmospheric research 51: 245-265.

57. Buechler DE, Driscoll TE, Goodman SJ, Christian HJ (2000) Lightning activity within a tornadic thunderstorm observed by the optical transient detector (OTD). Geophysical Research Letters 27: 2253-2256.

58. Anderson FJ, Freier GD (1974) Relative amounts of positive or negative charge in a lightning flash Journal of Geophysical Research 79: 5057-5058.

59. Williams E, Mushtak V, Rosenfeld D, Goodman S, Boccippio D (2005) Thermodynamic conditions favorable to superlative thunderstorm updraft, mixed phase microphysics and lightning flash rate. Atmospheric Research 76 288-306.

60. Nishihashi M, Arai K-i, Fujiwara C, Mashiko W, Yoshida S, et al. (2015) Characterization of lightning jumps associated with a tornadic supercell on 2 September 2013. SOLA. 1: 18-22.

61. Strader SM, Ashley WS (2014) Cloud-to-ground lightning signatures of longlived tornadic supercells on 27-28 April 2011. Physical Geography 35: 273-296.

62. Rudlosky SD, Fuelberg HE (2013) Documenting storm severity in the midAtlantic region using lightning and radar information. Monthly Weather Review 141: 3186-3200.

63. McCaul Jr EW, Cohen C (2002) The impact on simulated storm structure and Intensity of variations in the mixed layer and moist layer depths. Monthly Weather Review 130: 1722-1748.

\section{OMICS International: Publication Benefits \& Features}

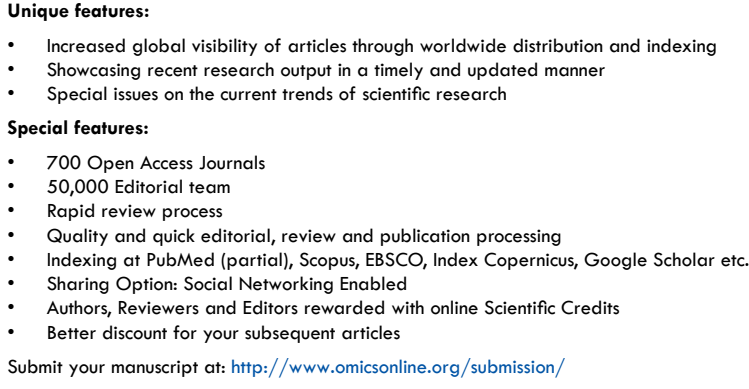

